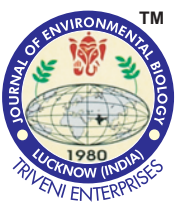

\title{
Morphometrics using radiographic study of thyroid cartilage for age-estimation in Korean males
}

\begin{abstract}
Authors Info
D.S. Kim, J.N. Park, Y.H. Yu, J.H. Kang, K.H. Lee, D.Y. Yoo and D.K. Park*

Department of Anatomy, Soonchunhyang University, Cheonan, 31151, Republic of Korea

*Corresponding Author Email : mdeornfl@sch.ac.kr
\end{abstract}

\section{Edited by}

Professor Seongho Ryu

\section{Reviewed by}

Professor Yi-Suk Kim

Professor U-Young Lee

\section{Abstract}

Aim: To estimate the age at death of Koreans whose age is unknown, based on the radiographic analysis of thyroid cartilage.

Methodology: Thyroid cartilage was separated from the larynx and dissected from the surrounding connective tissue. Dedicated mammography was carried out in 124 thyroid cartilage specimens, including 76 males and 48 females. Radiographed films were scanned and 17 quantitative measurements were carried out with Adobe ${ }^{\varpi}$ Photoshop ${ }^{\circledR}$ CS (version 8.0). These measurements were analyzed using the SPSS (version 22.0) statistical software package.

\begin{abstract}
Results: The data were divided into three age groups: G1- age between 15 and 30 years; G2- age between 31 and 45 years; G3- age over 46 years. The discriminant functions for the age group and multiple regression functions for each age group were obtained for the ageestimation of male subjects, but the application for female subjects was still limited due to small sample size for each age group.
\end{abstract}

Interpretation: The study indicates that thyroid cartilage is useful in estimating the age for Korean male subjects, whose age is unknown. Further investigations must be conducted to verify the utility of this method and its application to female subjects.

Key words: Age estimation, Korean males, Mammography, Radiographic analysis, Thyroid cartilage

How to cite : Kim, D.S., J.N. Park, Y.H. Yu, J.H. Kang, K.H. Lee, D.Y. Yoo and D.K. Park: Morphometrics using radiographic study of thyroid cartilage for ageestimation in Korean males. J. Environ. Biol., 40, 854-860 (2019). DOI : http://doi.org/10.22438/jeb/40/5(SI)/SI-05 


\section{Introduction}

The need for accurate methods for age estimation has increased in the last decade. The reasons for this increase are the increasing number of unidentified victims and the increase in cases requiring age estimation in living individuals with no proof of date of birth (Ritz-Timme et al., 2000). Most of the previous studies on age estimation have focused on using hard tissue such as teeth and bones. However, few studies have been carried out on the use of soft tissue such as cartilages.

Larynx extends from tongue to trachea, and controls the transfer of air and phonation with the tongue. The skeletal framework of larynx consists of several cartilages, and thyroid cartilage is the largest among the larynx cartilages. Thyroid cartilage consists of two quadrilateral laminae, and forms laryngeal prominence because the anterior parts of the laminae are fused together.

It is known that the component part of laryngeal cartilage changes with aging, because ossification of thyroid cartilage in males begins at a younger age than females. Roncallo (1948) reported that ossification involves more cartilage in males as compared to females. According to the radiological and histological study of the laryngeal cartilage by Keen and Wainwright (1958), while ossification in females is slower than males, it is difficult to presume age from the ossification progress. Also, they reported that the ossification method of laryngeal cartilage involves endochondral ossification, therefore calcification disappeared as endochondral ossification proceeded. Several radiological studies for the laryngeal cartilage of Western populations have reported that calcification and ossification change depending on the sexual dimorphism and age (Hately et al.,1965; Ajmani et al., 1980; Curtis et al., 1985; Turk and Hogg, 1993; de la Grandmaison et al., 2003).

Concerning Eastern populations, Matsuyama (1959) reported the age-onset of calcification and ossification, and the changes of ossification patterns in the thyroid cartilage of Japanese subjects. Sugiyama et al. (1995) suggested an equation that could estimate the age by measuring the ossification degree of thyroid cartilage in Japanese females. Cho (1962) described the detailed components of first appearance of calcification and ossification of thyroid cartilage in Korean, changes of the spreading and patterning of calcification and ossification with aging.

Although these studies have recognized that calcification and ossification in laryngeal cartilage changes depending on sexual dimorphism and age, quantitative analysis of the results are lacking. The purpose of this study was to estimate the age at death of Koreans whose age is unknown, based on the radiographic analysis of thyroid cartilage.

\section{Materials and Methods}

Subjects: One hundred and twenty four thyroid cartilage specimens (Table 1), including 76 males and 48 females were obtained from the Anatomy Laboratory, College of Medicine, the Catholic University of Korea and postmortem autopsies were conducted at the National Institute of Scientific Investigation. The specimens of thyroid cartilages were fixed in formalin.

Radiographic imaging: Thyroid cartilages were separated from the larynx and dissected from the surrounding connective tissue with care. Thyroid cartilages were then divided into right and left halves along their anterior border. 4-pairs of divided thyroid cartilages were arranged on AD-M (Fujifilm, Japan) film with their outer surfaces facing upwards. Dedicated mammography (Atomscope35M, MIKASA, Japan) was carried out on the radiographing condition of five Masses at $25 \mathrm{Kvps}$ (Fig. 1). The radiographed films were scanned (Epson Expression 1680 pros, Seiko Epson, Japan) and digitalized by resolution $300 \mathrm{dpi}$.

Quantitative analysis of calcification and ossification: The quantitative analysis of digitalized radiographs were carried out withAdobe $®$ Photoshop $®$ CS (Ver 8.0, Adobe, USA) computer program. The whole process was devided into five steps. The words within the quotation marks in five steps denote menus for performing tasks at the menu bar in the Adobe $₫$ Photoshop $®$ program.

Standardization of picture size: As canned radiograph was opened in the program, one thyroid cartilage among four-pairs was selected and changed the mode to "Indexed color". This image was saved as 'Sorted data' (Fig. 2A). In order to maintain standard size, all images were adjusted picture size to $1024 \times 1024$ pixels by "Canvas size". The image was saved as 'Resized data'(Fig. 2B).

Estimation of total area of thyroid cartilage: A 'Resized data' image was opened in the program and the whole area of cartilage was filled with white color using "Brush tool". After this process, the 'Resized data' image was renamed as 'Total area' (Fig. 2C). By clicking "Histogram" under "Window" on the menu bar, the pixels of the white area was counted. Therefore, the total pixels of thyroid cartilage of one half side was determined (Total area).

The distinction between calcification and ossification: $A$ 'Resized data' image was opened in the program and filled the rest area of cartilage with black color using "Brush tool". After this process, the 'Resized data' image was renamed as 'Original data' (Fig. 2D). By clicking"Eyedropper tool"on the toolbox, the density of each region is shown at the color picker as number between 0 and 255. We could distinguish the density of cartilage itself is below 45 , the density of ossification region is between 46 and 112 , and the density of calcification region was more than 113 by this process. 
Table 1: Number of thyroid cartilages

\begin{tabular}{llll}
\hline Age & Male & Female & 0 \\
\hline$\sim 10$ & 2 & 5 & 0 \\
$11 \sim 20$ & 5 & 6 & 0 \\
$21 \sim 30$ & 8 & 5 & 0 \\
$31 \sim 40$ & 9 & 8 & 0 \\
$41 \sim 50$ & 18 & 8 & 0 \\
$51 \sim 60$ & 15 & 4 & 0 \\
$61 \sim 70$ & 14 & 1 & 0 \\
$71 \sim$ & 5 & 15 & 0 \\
Total & 76 & 48 & 0 \\
\hline
\end{tabular}

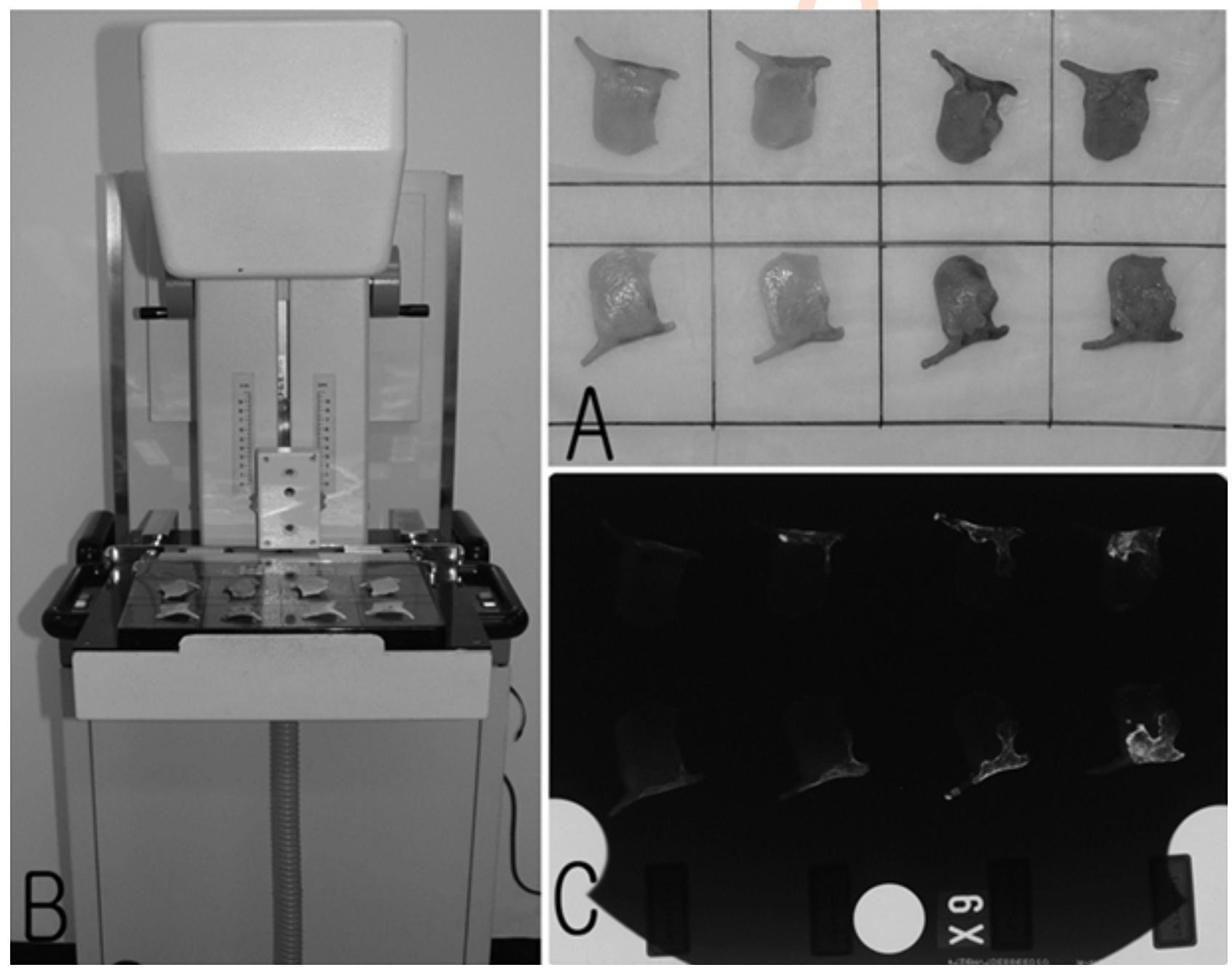

Fig. 1 : Photographs of the radiographic process using Atomscope35M, dedicated mammographic system. (A) Photograph showing example of radiography. (B) The arrangement of the specimens with their outer surfaces being faced upwards. (C) Scanned data of X-ray film. In the case of using an Image analyzer, a 25 cent coin was also radiographed with the specimen as a scale.

Area of calcification and ossification of thyroid cartilage: Owing to step (3), we could distinguish calcification and ossification. When we clicked "Threshold" under "Image" on the menu bar after an 'Original data' image was opened in the program, a dialog box will come up. If we typed 46 at a dialog box, then the region of cartilage disappeared. However, the regions of calcification and ossification remained, this image was renamed as 'Radio-opaque area' (Fig. 2E). If we typed 113 at a dialog box, 

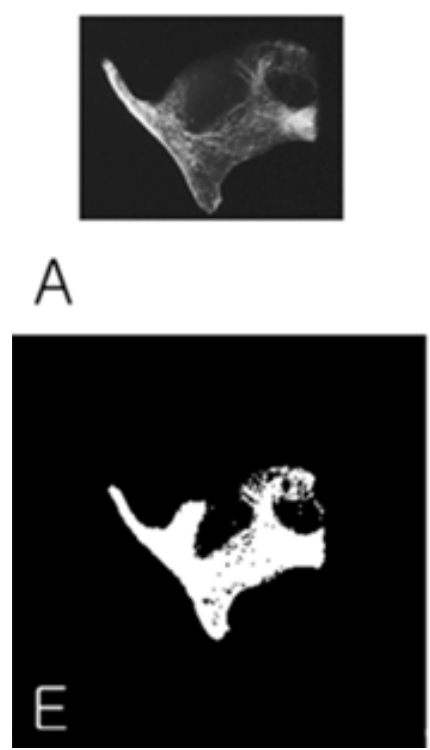
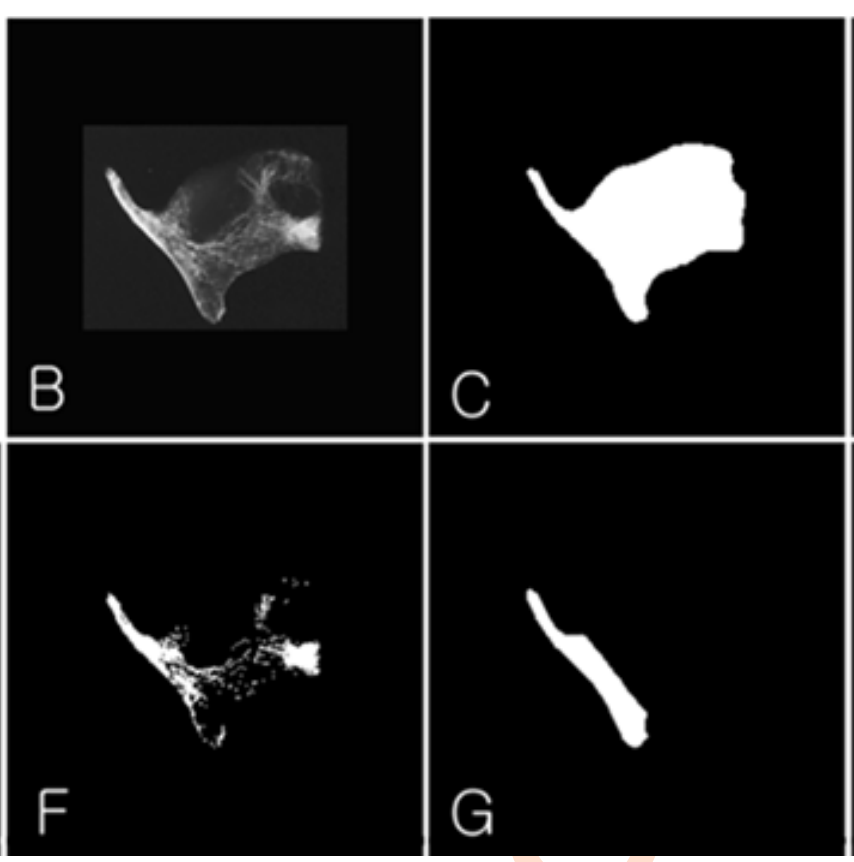
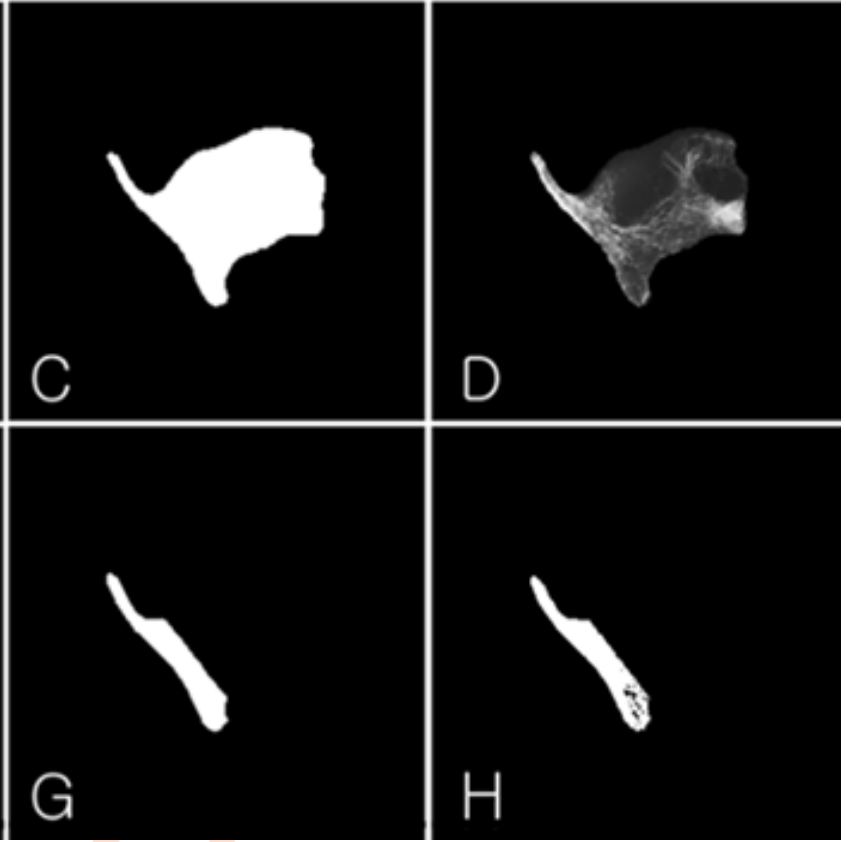

Fig. 2 : Footsteps for image processing of X-ray photographs using Adobe $₫$ Photoshop $₫$ CS. All words between within the quotation marks are menus

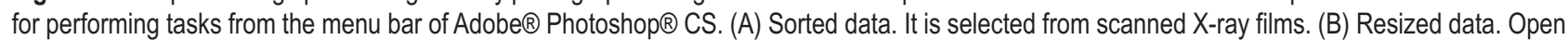
'Sorted data', choose "Image" and select "Canvas size", then resize the canvas as 1024×1024 pixels. (C) Total area. Open 'Resized data', fill the thyroid cartilage with white color, and the rest with black color. (D) Original data. Open 'Total area', choose "Magic wand" at the black background and "Copy" the selected area, then "Paste" it to 'Resized data'. (E) Radio-opaque areas. Open 'Original data', choose "Image" and select "Adjustments", then "Threshold" as 46. (F) Calcification area. Open 'Original data', click "Image" and select "Adjustments", then "Threshold" as 113. (G) Total Horn area. Open 'Original data', fill the laminae of the thyroid cartilage with black color, and the rest with white color. (H) Horn radio-opacity. Open 'Total Horn area', choose "Magic wand" on the black background and "Copy" the selected area, then "Paste" it to 'Original data'. Then select "Adjustments", then "Threshold" as 46.

then the region of cartilage and ossification disappeared. However, the regions of calcification remained only, this image was renamed as 'Calcification area' (Fig. 2F). By clicking "Histogram" under "Window" on the menu bar, the pixels of 'Radio-opaque area' and 'Calcification area' could be counted. Therefore, we could know the total pixels of ossification and calcification in thyroid cartilage. In order to calculate the ratio for ossification and calcification, the number of pixels of ossification or calcification was divided by the number of pixels in the 'Total area'.

Area and degree of radio-opacity in horn region: A 'Total area' image was opened in the program and filled the lamina of cartilage with black color using "Brush tool". After this process, the 'Total area' image was renamed as 'Total Horn area' (Fig. 2G). By clicking "Histogram" under "Window" on the menu bar, the pixels of 'Total Horn area' was counted. A 'Resized data' image was opened in the program and filled the lamina of cartilage with black color using "Brush tool". When we clicked "Threshold" under "Image" on the menu bar after a 'Resized data' image was opened in the program, a dialog box will come up. If we typed 46 at a dialog box, then the region of cartilage disappeared. This image was renamed as 'Horn radio-opacity' (Fig. 2H). By clicking
"Histogram" under "Window" on the menu bar, the pixels of 'Horn radio-opacity' could be counted. Therefore, we could know the total pixels of horn area and ossification in horn area. In order to calculate the ratio for ossification and calcification, the number of pixels of 'Horn radio-opacity' was divided by the number of pixels in the 'Total Horn area'.

Seventeen quantitative measurements (Table 2) were carried out using Adobe $®$ Photoshop $®$. Statistical analysis was performed on 17 quantitative measurements (Table 2) using the SPSS (version 22.0) statistical software package.

\section{Results and Discussion}

For relevant statistical analysis, the data were divided into four age groups: Group 0- age below 14 years; Group 1- age between 15 and 30 years; Group 2-age between 31 and 45 years; and Group 3-age over 46 years.

The total Pixels of thyroid cartilage (TP, either half side) showed significant differences among the age groups and between the sexes. The means of TP of Group 1,2 and 3 in both sexes exhibited larger values than those of Group 0 in both sexes $(p<0.01)$. The means of TP of Groups 1,2 and 3 in male subjects 
Total pixels of thyroid cartilage (TP) of one half side

(Excluding the age under 14 years by $\mathrm{TP}<62,000$ )
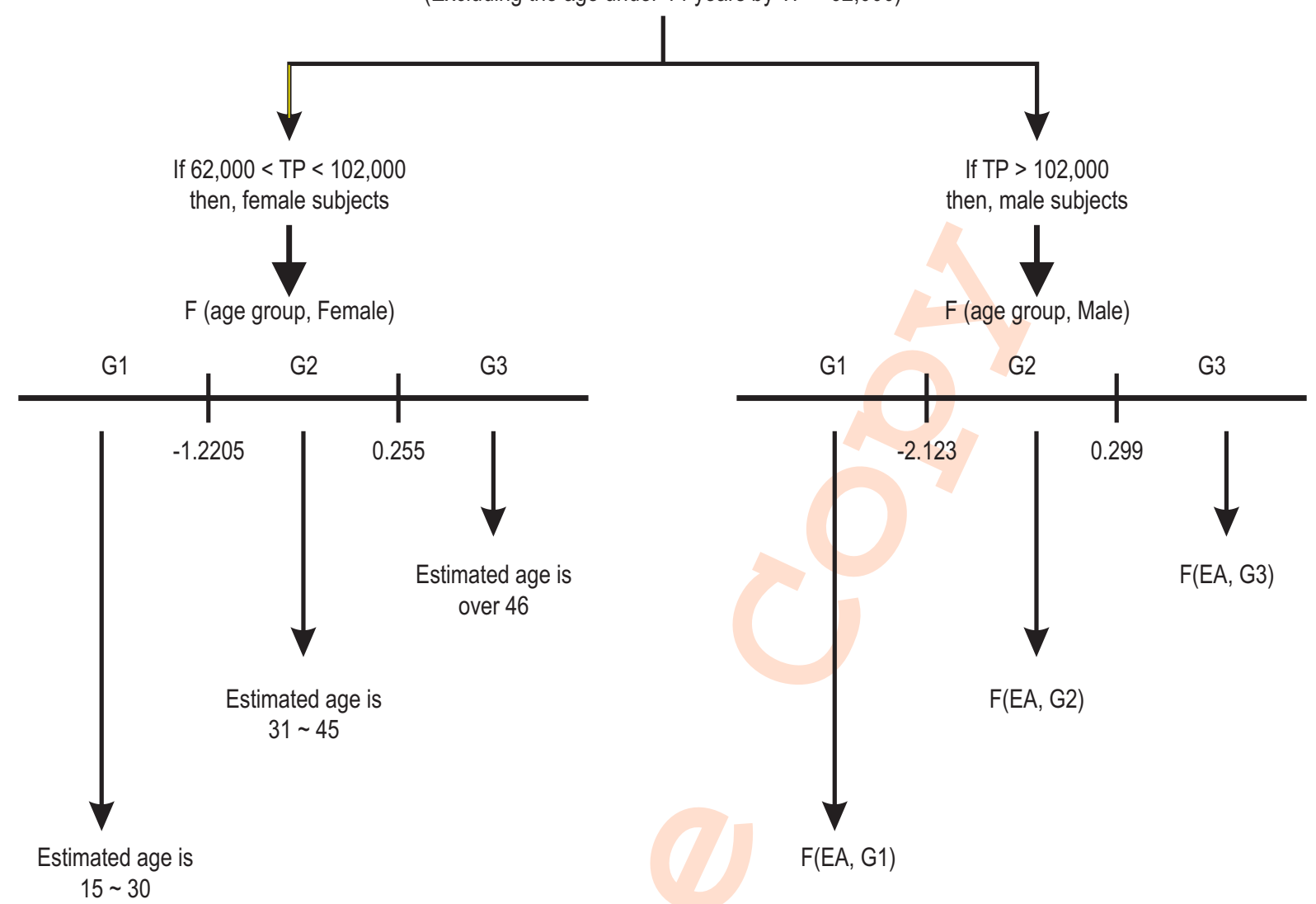

Fig. 3 : Flowchart of age-estimation of unknown corpse using Adobe $®$ Photoshop $®$ CS. Initially, the possibility of age under 14 years was excluded according to the total pixels of thyroid cartilage (TP) of one-half side. Also according to the numbers of TP, male subjects were distinguished from female subjects. In the case of female subject, groups were allocated for age-estimation by age-grouping discriminant functions for female subjects. In the case of a male subject, age-estimation was possible by multiple regression functions for each age group, followed by age-grouping discriminant functions for male subjects. $\mathrm{G} 1$ = Group 1, age between 15 and 30 years ; $\mathrm{G} 2=$ Group 2, age between 31 and 45 years; $\mathrm{G} 3=$ Group 3, age over 46 years; $F=$ function ; $E A=$ estimated age.

exhibited larger values than those of Groups 1,2 and 3 in female subjects $(p<0.01)$. In summary, if the number of TP was less than 62,000 , a subject might be younger than 14 years regardless of sex; if the number of TP was between 62,000 and 102,000, a subject might be female older than 15 years; if the number of TP was greater than 102,000 , a subject might be male older than 15 years. Therefore, the pixel numbers of 62,000 could be demarcation values for age, and the pixel numbers of 102,000 could be the demarcation values for sex.

In order to estimate the age in male subjects, 17 measurements (Table 2 ) were statistically analyzed. The results of oneway ANOVA for male subjects are listed in Table 3 and 4. Fifteen measurements increased with age. The flow chart for ageestimation process is shown in Fig. 3.

To investigate an unidentified victim who's body is badly decomposed or found in dried bones, biological profiles such as ethnicity, sex, age and stature need to be determined. Among them, the need for accurate methods for age estimation increased due to increase in the number of unidentified victims (Ritz-Timme et al., 2000).

Forensic studies for the estimation of age at death was carried out by focussing on hard tissue such as teeth or bones. Teeth are generally applied for age estimation, because deciduous and permanent teeth have different age-onset of eruption and exfoliation, and permanent teeth, without injury or disease, share common morphological development processes after eruption. The development process of teeth (Garn et al., 1959 ; Lewis and Garn, 1960 ; Anderson et al., 1976) can be used as a criterion for the estimation of age before adolescence. In the adult, histological investigations of teeth (Gustafson, 1950 ; Maples, 1978 ; Lamendin et al., 1992) and the study of enamel layer abrasion (Miles, 1962) can be adopted. For age estimation with bone, the degree of cranial suture closure with ossification (Todd and Lyon, 1924, 1925a, 1925b, 1925c; Meindland Lovejoy, 1985) and alterations of symphyseal surface of the pubis with 
Table 2 : List of measurements of ossification or calcification in thyroid cartilage

\begin{tabular}{ll}
\hline No & Measurement \\
\hline 1,2 & Total Pixels of Thyroid Cartilage(Rt. \& Lt.) \\
3,6 & Ratio of Ossification of Thyroid Cartilage(Rt. \& Lt.) \\
4,7 & Ratio of Calcification of Thyroid Cartilage**(Rt. \& Lt.) \\
5,8 & Ratio of Opacity of Thyroid Cartilage*(Rt. \& Lt.) \\
9,11 & Ratio of Opacity of horn**(Rt. \& Lt.) \\
10,12 & Degree of Opacity of horn(Rt. \& Lt.) \\
13 & Sum of Ratio of Rt. \& Lt. Ossification(3+6) \\
14 & Sum of Ratio of Rt. \& Lt. Calcification(4+7) \\
15 & Sum of Ratio of Rt. \& Lt. Opacity(5+8) \\
16,17 & Ratio*Degree of Opacity of horn(Rt. \&Lt.)(9*10 \& 11*12) \\
\hline
\end{tabular}

= Pixels of "Threshold 46"/ total pixels of thyroid cartilage;" = Pixels of "Threshold 113"/ Total Pixels of Thyroid Cartilage; "" = Length of opacity in horn area/ Length of horn

Table 3: Measured values of the thyroid cartilage of male subjects

\begin{tabular}{llllll}
\hline No & Measurement & Group 0 (SD) & Group 1 (SD) & Group 2 (SD) & Group 3(SD) \\
\hline 1 & Total Pixels, Rt. & $58858(3897.6)$ & $140556.2(14854.0)$ & $150350.5(14281.4)$ & $145646.3(20153.6)$ \\
2 & Total Pixels, Lt. & $58177.5(1287.9)$ & $140286.9(14031.3)$ & $151904.7(14914.3)$ & $147894.8(18513.6)$ \\
3 & Ratio of Ossification, Rt. & $0.0(0.0)$ & $9.1(8.5)$ & $26.9(11.2)$ & $33.2(15.5)$ \\
4 & Ratio of Calcification, Rt. & $0.0(0.0)$ & $1.1(1.6)$ & $9.4(9.6)$ & $21.9(12.0)$ \\
5 & Ratio of Opacity, Rt. & $0.0(0.0)$ & $10.2(9.0)$ & $36.4(19.3)$ & $55.1(19.7)$ \\
6 & Ratio of Ossification, Lt. & $0.0(0.0)$ & $7.8(8.5)$ & $23.0(13.2)$ & $33.5(17.0)$ \\
7 & Ratio of Calcification, Lt. & $0.0(0.0)$ & $0.8(1.2)$ & $6.1(6.6)$ & $17.4(9.7)$ \\
8 & Ratio of Opacity, Lt. & $0.0(0.0)$ & $8.6(8.6)$ & $29.0(19.1)$ & $50.9(19.8)$ \\
9 & Ratio of Opacity of Horn, Rt. & $0.0(0.0)$ & $16.5(16.6)$ & $57.5(23.3)$ & $81.0(14.8)$ \\
10 & Degree of Opacity of Horn, Rt. & $0.0(0.0)$ & $0.3(0.2)$ & $0.7(0.2)$ & $1.0(0.1)$ \\
11 & Ratio of Opacity of Horn, Lt. & $0.0(0.0)$ & $15.0(17.4)$ & $49.7(23.5)$ & $75.3(16.5)$ \\
12 & Degree of Opacity of Horn, Lt. & $0.0(0.0)$ & $0.3(0.2)$ & $0.7(0.2)$ & $1.0(0.1)$ \\
13 & Sum of Rt. \& Lt. Ossification & $0.0(0.0)$ & $16.9(16.9)$ & $49.9(23.9)$ & $66.7(31.6)$ \\
14 & Sum of Rt. \& Lt. Calcification & $0.0(0.0)$ & $1.9(2.8)$ & $15.5(15.8)$ & $39.2(20.6)$ \\
15 & Sum of Rt. \& Lt. Opacity & $0.0(0.0)$ & $18.8(17.5)$ & $65.4(37.7)$ & $106.0(39.1)$ \\
16 & Ratio*Degree of Opacity of Horn, Rt. & $0.0(0.0)$ & $6.8(9.3)$ & $42.4(24.5)$ & $78.7(17.3)$ \\
17 & Ratio*Degree of Opacity of Horn, Lt. & $0.0(0.0)$ & $6.6(9.6)$ & $39.4(26.2)$ & $73.0(19.2)$ \\
\hline
\end{tabular}

aging (Todd, 1920, 1921 ; Gilbert and Mckern, 1973 ; Suchey, 1979 ; Suchey et al., 1979 ; Angel et al., 1986 ; Suchey et al., 1986) can be utilized.

Radiographic studies of costochondral region (Barres et al., 1989) and larynx cartilages were performed. These studies revealed that the degree of calcification or ossification in these cartilages increased with the aging process; however, quantitative analysis was lacking and the results are therefore limited to use as criteria for age estimation.

In this study, we performed quantitative analysis of calcification and ossification of thyroid cartilage (using Adobe $\circledR$ Photoshop $®$ CS that is a commercialized computer program), and confirmed that the average values of ossification, calcification, and opacity in the cartilage increased with age in both sexes, which corresponded to the previous findings. The difference of TP (either half side) values, depending on age and sex, were very distinct that it was possible to distinguish three groups (a male or female subject under 14 years old, a female subject over 15 years old, and a male subject over 15 years old).Also, in both sexes, the values of calcification, opacity and opacity in the horn were larger on the right side than on the left side, so further investigations for this etiology are required.

The multiple regression, in which the age as Y-axis and the measured items as $X$-axis, was the best statistical analysis method for verifying the above results; however, the acquired standard deviation was 15 years, and this function was not able to be applied for age estimation(Ritz-Timme et al., 2000). Therefore, to acquire results from the multiple regression that are more meaningful, the results of age group 0 (under 14 years old) in both sexes were excluded. The remainder, divided into group 1, 2 and 3 , were analyzed with the canonical discriminant. Eight measurements among the total of 17 measurements (Table 2) were available for discrimination for the age group. The acquired 
age-grouping discriminant function for male subjects are as follows:

$F$ (age group, Male $)=0.063^{*} \times 1-0.024^{*} \times 2-0.05^{*} \times 3+0.011^{*} x 4-$ $3.968^{*} x--0.05^{*} x 6+10.096^{*} x 7+0.018^{*} x 8-4.805$

X1 :No. 16 (Ratio * Degree of Opacity of horn, Right); X2 :No. 17 (Ratio * Degree of Opacity of horn Left); X3 :No. 15 (Sum of Right \&Left Opacity); X4 :No. 14 (Sum of Right \&Left Calcification); X5 :No. 12 (Degree of Opacity of Horn, Left); X6 :No. 11 (Ratio of Opacity of Horn, Left); X7 :No. 10 (Degree of Opacity of Horn, Right); X8 :No. 9 (Ratio of Opacity of Horn, Right)

When the calculated value by this formula was lower than -2.123 , the subject was classified as belonging to age group 1 (15 and 30 years old), when the value was between -2.123 and 0.299 , the subject was classified as belonging to age group 2 (31 45 years old), and when the value was higher than 0.299 , the subject was classified as belonging to age group 3 (over 46 years old). The hit ratio of function was $87.8 \%$.

The results of this radiographic study of thyroid cartilage in Korean population will be useful in the case of male subjects for estimating the age at death, if an unidentified cadaver with thyroid cartilage was found. However, in the case of female subjects, the results can only be applied for age-grouping. Also, radiographic study of thyroid cartilage can reduce the amount of time spent on age-estimation, which required more time when teeth were used to determine the age.

\section{Acknowledgment}

This work was supported by the Soonchunhyang University.

\section{References}

Anderson, D.L., G.W. Thompson and F. Popovich: Age of attainment of mineralization stages of the permanent dentition. J. Forensic Sci., 21, 191-200(1976).

Angel, J.L., J.M. Suchey, M.Y. İscan and M.R. Zimmerman: Age at death estimated from the skeleton and viscera. In : Dating and age determination of biological materials (Eds.: M.R. Zimmerman and J.L. Angel). Croom Helm, London, U.K., p. 179-220 (1986).

Ajmani, M.L., S.P. Jain and S.K. Saxena: A metrical study of laryngeal cartilages and their ossification. Anat. Anz.,148, 42-48(1980).

Barrés, D.R., M. Durigon and F. Paraire: Age estimation from quantitation of features of "chest plate" X-rays. J. Forensic Sci., 34, 228-33 (1989).

Curtis, D.J., R.M. Allman, J. Brion, G.S. Holborow and S.L. Brahman: Calcification and ossification in the arytenoid cartilage: Incidence and patterns. J. Foren. Sci., 30, 1113-1118(1985).

de la Grandmaison, G.L., A. Banasr and M. Durigon: Age estimation using radiographic analysis of laryngeal cartilage. Am. J. Forensic Med. Pathol., 24, 96-99 (2003).

Garn, S.M., A.B. Lewis and D.L. Polacheck: Variability of tooth formation. J. Dent. Res., 38, 135-48 (1959).
Gilbert, B.M. and T.W. McKern: A method for aging the female Os pubis. Am. J. Phys. Anthropol., 38, 31-8 (1973).

Gustafson, G.: Age determination on teeth. J. Am. Dent. Assoc., 31, 4554 (1950).

Hately, W., G. Evison and E. Samuel: The pattern of ossification in the laryngeal cartilages : A radiographic study. Br. J. Radiol., 38, 585591 (1965)

Keen, J.A. and J. Wainwright: Ossification of the thyroid, cricoid and arytenoid cartilages. S. Afr. J. Lab. Clin. Med., 4, 83-108 (1958).

Lamendin, H., E. Baccino, J.F. Humbert, J.C. Tavernier, R.M. Nossintchouk and A. Zerilli: A simple technique for age estimation in adult corpses. The two criteria dental method. J. Forensic Sci., 37, 1373-9 (1992).

Lewis, A.B. and S.M. Garn: The relationship between tooth formation and other maturational factor. Angle. Orthod., 30, 70-77 (1960).

Maples, W.R.: An improved technique using dental histology for estimation of adult age. J. Forensic Sci.,23, 764-70 (1978).

Matsuyama, Y.: Physical anthropological study on the thyroid in Kuju Japanese. Japanese J. ENT,62, 899-924 (1959).

Meindl, R.S. and C.O. Lovejoy: Ectocranial suture closure: A revised method for the determination of skeletal age at death based on the lateral-anterior sutures. Am. J. Phys. Anthropol., 68, 57-66 (1985).

Miles, A.E.: Assessment of the ages of a population of Anglo-Saxons from their dentition.Proc. R. Soc. Med., 55, 881-866(1962).

Ritz-Timme, S., C. Cattaneo, M.J. Collins, E.R. Waite, H.W. Schütz, H.J. Kaatsch and H.I. Borrman: Age estimation: The state of the art in relation to the specific demands of forensic practice. Int. J. Legal Med.,113, 129-136(2000).

Sugiyama, S., S. Tatsumi, H. Noda and M.Yamaguchi.: Estimation of age from soft X-ray findings of Japanese female thyroid cartilages. Jpn. J. Legal Med., 49, 236-241(1995).

Suchey, J.M.: Problems in the aging of females using the Os pubis. Am. J. Phys. Anthropol., 51, 467-70(1979).

Suchey, J.M., D.V. Wiseley, R.F. Green and T.T. Noguchi: Analysis of dorsal pitting in the os pubis in an extensive sample of modern American females. Am. J. Phys. Anthropol., 51, 517-40(1979).

Suchey, J.M., D.V. Wiseley and D. Katz: Evaluation of the todd and mckern-stewart methods for aging the male os pubis. In : Forensic Osteology (Ed.: K.J. Reichs). Charles C. Thomas Springfield, FL., pp. 33-67(1986).

Todd, T.W.: Age changes in the pubic bone: I, The male white pubis. Am. J. Phys. Anthropol., 3, 285-334(1920).

Todd, T.W.: Age changes in the pubic bone. Am. J. Phys. Anthropol., 4, 170 (1921).

Todd, T.W. and D.W. Jr Lyon: Endocranial suture closure: Its progress and age relationship. Part I, Adult males of white stock. Am. J. Phys. Anthropol., 7, 325-384(1924).

Todd, T.W. and D.W. Jr Lyon: Cranial suture closure: Its progress and age relationship. Part II, Ectocranial closure in adult males of white stock. Am. J. Phys. Anthropol., 8, 23-45 (1925a).

Todd, T.W. and D.W. Jr Lyon: Cranial Suture Closure: Its progress and age relationship. Part III, Endocranial closure in adult males of nigro stock. Am. J. Phys. Anthropol., 8, 47-71(1925b).

Todd, T.W. and D.W. Jr Lyon: Cranial suture closure: Its progress and age relationship. Part IV, Ectocranial closure in adult males of nigro stock. Am. J. Phys. Anthropol., 8, 149-168 (1925c).

Turk, L.M. and D.A. Hogg: Age changes in the human laryngeal cartilages. Clin. Anat., 6, 154-162 (1993). 